\title{
Evolution of LTCC technology for industrial applications
}

\author{
Michel Massiot \\ CMAC Microtechnology, 44 Av de la Glacière, Argenteuil, michelmassiot@cmac.com \\ The LTCC (Low Temperature Cofired Ceramic) technology has been used for years in various applications \\ like automotive (under the hood), data-processing, telecoms, datacoms, military and space, industrial, \\ packaging. The evolution of the materials, processes and approach have reinforced its cost and performance \\ attractivity. This is particularly true in the case of high frequency / optoelectronic products. This paper gives \\ an overview of the enabling features of the LTCC technology for these applications, as well as an example of \\ a $10 \mathrm{~Gb} / \mathrm{s}$ transmitter.
}

\section{INTRODUCTION}

New needs exist in the high frequency range (5 to $77 \mathrm{GHz}$ ! ) in markets such as telecom / datacoms, military, space and automotive, for example broadband wireless or optoelectronic integrated modules applications. LTCC is the technology of choice for those applications, thanks to a number of enabling features, which are summarized below.

\section{LTCC BASICS}

The LTCC is now widely known, however not so much as organic PCB. So the general concept is reminded hereafter. The result is a monolithic ceramic multilayer :

\section{LTCC GENERAL HIGH FREQUENCY FEATURES}

The LTCC technology has per nature a number of interesting features for high frequency applications:

- Very good electrical characteristics and stability up to millimeter waves. This is particularly important for adapted lines for example. In addition to the inherent properties of the materials, the LTCC is insensitive to moisture, which is known to influence and degrade largely many organic compositions. Depending on the most common material systems used, dielectric constants are between 6 and 9 in the $\mathrm{GHz}$ range, loss tangent between 0.001 and 0.006 . The use of $\mathrm{Ag}$ printed conductors contributes to these performances.

- - Outstanding dimensional and mechanical stability. This is due to the glass-ceramic nature of LTCC. Not only the TCE is low (5 to 7 $\mathrm{ppm} /{ }^{\circ} \mathrm{C}$ ), but the thermomechanical properties have a elastic,

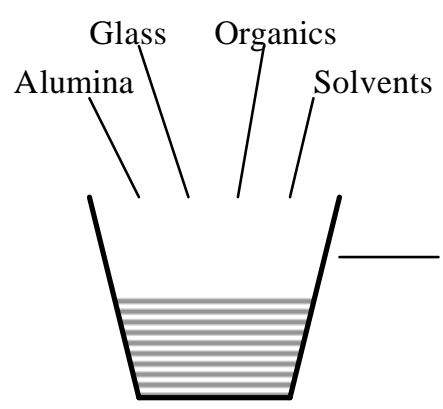

Slurry preparation

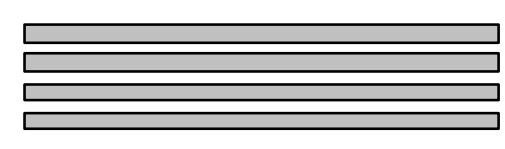

Tape casting (several layers represented)

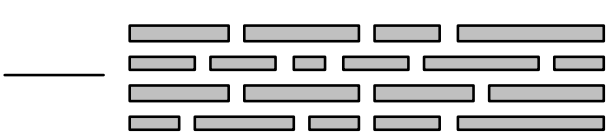

Punching

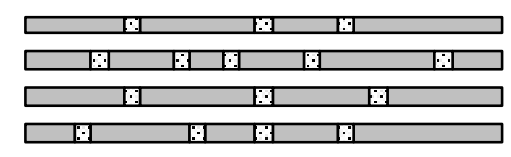

Via filling and conductor printing

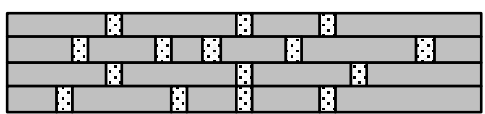

Stacking, pressing, cofiring 
reversible behavior largely beyond the use temperature range.

-Low TCE: this is also a great avantage of ceramics. It is close to semi-conductor ( $\mathrm{Si}, \mathrm{GaAs}$, InP) TCEs, facilitating their use in chip \& wire or flip-chip form. Note that the LTCC is very adapted to short wedge-wedge bonding, thanks to the patterning and machining precision ability. Its TCE is also in the range of glass fibers, allowing for maintaining better alignment over temperature span.

-Good thermal conductivity: although the LTCC dielectrics have only moderate intrinsic thermal conductivity value ( 2 to $4 \mathrm{~W} / \mathrm{m} .{ }^{\circ} \mathrm{C}$ typically), it is far better than organic PCBs. Also it can be largely improved with the use of thermal vias (up to 50 $\left.\mathrm{W} / \mathrm{m} .{ }^{\circ} \mathrm{C}\right)$.

-Fine lines patterning : either by direct printing or using photoimageable means

-3D capability: ease to produce cavities, holes, fences

-Hermeticity and brazing possibilities

-Buried passive components and functions, trimmability...

Additionally to this, the technology has proven reliability and cost-effectiveness in demanding applications like ABS or Bluetooth.

\section{EVOLUTION OF NEEDS IN OPTOELECTRONIC APPLICATIONS \\ INDUSTRIAL}

The electro-optical interface is fast becoming the cornerstone of modern fixed-line communications networks, as operators move further towards an entirely optical infrastructure. At present, however, implementation of advanced optoelectronic modules is complicated by the fact that the broad range of capabilities required tends to be scattered among a diversity of IP (intellectual property) holders. Companies able to propose a full design, manufacture and test solution for integrated optoelectronic modules will be the preferred partners for telecommunications OEMs in the imple mentation of multichannel optical transmitters and receivers, photonic switches, array waveguide gratings, add-drop multiplexers, optical modulators and other advanced optoelectronic products.

These integrated modules have to be based upon a range of enabling technologies including optical fibre alignment and splicing, three-dimensional ceramic structures, chip-and-wire assembly and hermetic sealing. Again, in this full set of necessary portfolio, LTCC has a central position due to the above-mentioned features.

\section{EVOLUTION OF LTCC TECHNOLOGY}

From the above, it can be deduced that the effort will be put in the following directions :

-Materials : new formulations with ever better electrical characteristics, still being easily processable and adapted to complementary processes (brazing, wire-bonding...), specialties for more passive integration, optical specialties, all with acceptable cost,

-Processes : higher shrinkage control (through $\theta$ shrinkage or not), bigger processing size, finer lines, 3D processes improvement, better use of dissimilar materials

-Complementarity, vertical integration : a full range of associated technologies (fiber splicing, active alignment, laser hermetic sealing, thermal management, advanced die handling, test capability...) should be available at a single service supplier in order to simplify the life of the endusers and optimize the price of the products through global module co-design and manufacturing.

\section{APPLICATION EXAMPLE : SMALL FOOTPRINT - $10 \mathrm{~Gb} / \mathrm{s}$ TRANCEIVER}

The module shown below is an example of what the LTCC technology can offer in optoelectronics. It is a $10 \mathrm{~Gb} / \mathrm{s}$ tranceiver using LTCC as integral substrate and packaging part, with 3 different horizontal levels. Grooves are created in order to pre-align the fibers with the laser and the PIN detector. High speed electronics makes use of the electrical performances of LTCC and its feedthrough capabilities. The whole is hermetically sealed, leading to a highly integrated solution. This module is one member from a larger module family.

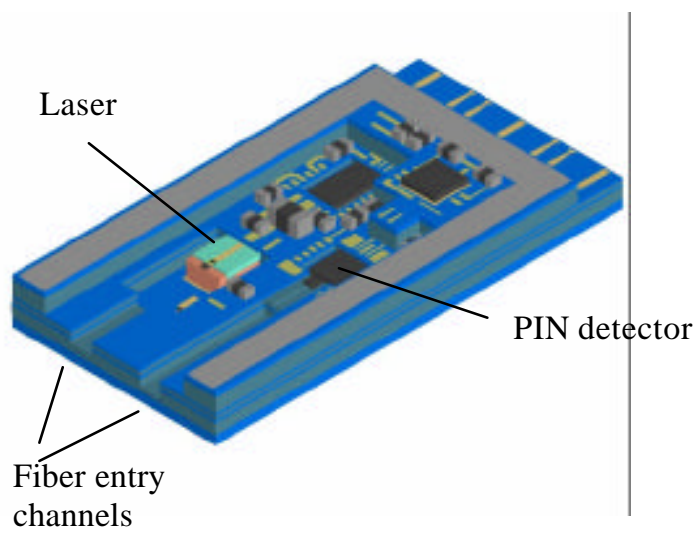

